\section{Conhecimento de preservativo masculino entre adolescentes: estudo de base populacional no semiárido nordestino, Brasil}

\author{
Knowledge of condom among adolescents: a \\ population-based study in the semiarid region of \\ Northeast Brazil
}

\author{
Conocimiento sobre preservativos entre los \\ adolescentes: estudio de base poblacional en la \\ región semiárida del Nordeste, Brasil
}

Juliana Mano Hartmann 1 Juraci A. Cesar ${ }^{1}$

\author{
1 Universidade Federal do Rio \\ Grande, Rio Grande, Brasil. \\ Correspondência \\ J. M. Hartmann \\ Universidade Federal do Rio \\ Grande. \\ Rua General Vitorino 80 \\ Rio Grande, RS 96200-310, \\ Brasil. \\ ju_hart@yahoo.com.br
}

\begin{abstract}
This study aimed to determine the prevalence of lack of knowledge on condoms and to identify associated factors among teenagers in two municipalities (counties) in Piauí State, Brazil, in mid-2008. Standardized home interviews were conducted by trained interviewers with all the teenagers (13-19 years of age). The study investigated demographic and socioeconomic characteristics and knowledge on contraception and sexually transmitted diseases (STDs). The outcome variable was lack of knowledge on male condoms. The multivariate analysis used Poisson regression with robust variance. Of the 2,241 adolescents, $18.8 \%$ claimed ignorance of male condoms. Among individuals with at least 9 years of schooling, only 4\% lacked knowledge on condoms. Meanwhile, among individuals who lacked knowledge on STDs, 74\% lacked knowledge on condoms. In the adjusted analysis, female gender, younger age, low schooling, not having a girlfriend, and lack of knowledge on oral contraception and STDs increased the likelihood of lack of knowledge on condoms. The data showed the urgent need for awareness-raising interventions on condom use among adolescents in these municipalities.
\end{abstract}

Condoms; Contraception; Sexually Transmitted Diseases; Adolescent

\section{Resumo}

Buscou-se determinar a prevalência e identificar fatores associados ao não conhecimento de preservativo masculino entre adolescentes, em dois municípios do semiárido piauiense, Brasil, em meados de 2008. Entrevistadores treinados aplicaram questionário padronizado a todos adolescentes (13-19 anos), nos domicílios. Investigaram-se características demográficas, socioeconômicas e conhecimento sobre contracepção e doenças de transmissão sexual (DST). O desfecho foi o não conhecimento de preservativo masculino. Na análise multivariada, utilizou-se regressão de Poisson com ajuste robusto da variância. Dos 2.241 adolescentes, 18,8\% disseram não conhecer preservativo. A prevalência de não conhecimento variou de 4\% para os com nove anos ou mais de escolaridade a 74\% entre os que não conheciam DST. Na análise ajustada, ser do sexo feminino, ter pouca idade, baixa escolaridade, não ter namorada, desconhecer contracepção oral e DST aumentava a probabilidade de não conhecer preservativo em relação às demais categorias. Os dados mostram a urgente necessidade de intervenção entre adolescentes desses municípios.

Preservativos; Anticoncepção; Doenças Sexualmente Transmissíveis; Adolescente 
Introdução

A Organização Mundial da Saúde (OMS) define adolescência como o período dos 10 aos 19 anos de idade, fase em que ocorre, geralmente, o início da vida sexual. Tal prática envolve pelo menos três atitudes de grande importância para os adolescentes: uso de método contraceptivo, ocorrência de gravidez e de doenças de transmissão sexual (DST) ${ }^{1}$. Por essa razão, lidar de forma adequada com contracepção é de fundamental importância tanto para prevenir gravidez quanto para a ocorrência de doenças de transmissão sexual.

A maioria dos métodos existentes pode ser utilizada pelos adolescentes, sendo os mais indicados o preservativo (condom ou camisinha), que além de não possuir contraindicação, auxilia na prevenção da gravidez e de doenças de transmissão sexual; o anticoncepcional oral e os injetáveis. Os demais métodos são pouco apropriados a essa faixa etária quer seja pela ocorrência de complicações, como é o caso do dispositivo intrauterino ou pela baixa efetividade, como os métodos comportamentais ou naturais (tabela ou calendário, curva basal, muco cervical e coito interrompido) 2 .

Os métodos contraceptivos mais amplamente conhecidos pelos adolescentes são preservativo masculino e anticoncepcional oral 3. A prevalência de conhecimento desses métodos variou de $84 \%$ a $99 \%$ para o preservativo e de $68 \%$ a $98 \%$ para o contraceptivo oral $1,3,4,5$. Esse conhecimento é maior entre adolescentes de maior idade, melhor escolaridade e elevada renda familiar 4,6,7.

Estudos sobre adolescentes são, em geral, restritos a grandes centros urbanos e em particular às escolas 8,9. Nessas áreas, por apresentarem nível socioeconômico elevado, o acesso dos adolescentes às informações sobre sexualidade é mais fácil, e o assunto pode ser tratado de forma mais aberta, o que facilita conhecê-lo. A restrição ao ambiente escolar decorre da facilidade em abordar grande número de adolescentes em um único lugar, o que confere rapidez à coleta de dados e reduz o custo do estudo.

O presente estudo foi conduzido em nível domiciliar no semiárido nordestino, Brasil, e teve por objetivo medir a prevalência e identificar fatores associados ao não conhecimento de preservativo masculino entre adolescentes residentes em dois municípios localizados no sul do Estado do Piauí.

\section{Métodos}

Este estudo foi conduzido no semiárido piauiense e faz parte de um projeto mais amplo cujo objetivo era reduzir a ocorrência de gravidez na adolescência. Para tanto, incluiu o Município de Caracol como o de intervenção e Anísio de Abreu como município-controle. Esses dois municípios estão localizados no sul do Estado do Piauí, a aproximadamente $600 \mathrm{~km}$ de Teresina, a capital. Na ocasião, Caracol possuía pouco mais de 10 mil habitantes, enquanto Anísio de Abreu cerca de 8,5 mil. A agricultura e o emprego público, sobretudo nas prefeituras locais, era base da economia desses municípios. O produto interno bruto (PIB) per capita de Caracol era de R $\$ 2.600$. O sistema local de saúde era formado por duas unidades básicas de saúde (UBS), sendo uma na zona rural e outra na zona urbana, e um pronto atendimento, todos pertencentes ao Sistema Único de Saúde (SUS). Em Anísio de Abreu, o PIB per capita somava R\$2,50 e havia três UBS, duas na zona rural e uma na zona urbana, além de um hospital de pequeno porte, todos pertencentes ao SUS. O Índice de Desenvolvimento Humano (IDH) era de 0,59 em Caracol e de 0,63 em Anísio de Abreu (Programa das Nações Unidas para o Desenvolvimento. http://www.pnud.org. br/atlas/ranking/IDH_Municipios_Brasil_2000. aspx?indiceAccordion=1\&li=li_Ranking2003, acessado em 06/Set/2012), e cerca da metade da população vivia abaixo da linha de pobreza (Instituto Brasileiro de Geografia e Estatística. http:/ / www.censo2010.ibge.gov.br/sinopse, acessado em 20/Jul/2012).

Os dados apresentados neste artigo são originários do estudo de linha de base realizado nesses municípios entre os meses de julho e setembro de 2008. Foram incluídos todos os adolescentes com idade entre 13 e 19 anos residentes nas áreas urbana e rural dos dois municípios. Decidiu-se por essa faixa etária em virtude de o estudo piloto mostrar que aproximadamente $90 \%$ dos adolescentes com idade entre 10 e 12 anos não se mostraram capazes de responder ao questionário autoaplicável. Essa faixa de idade é utilizada em vários estudos que tratam do tema. Por fim, adolescentes com limitação cognitiva importante ou institucionalizados não fizeram parte da amostra.

Este estudo censitário teve delineamento transversal 10 e cálculo de tamanho amostral feito a posteriori pela prevalência de não conhecimento de preservativo masculino de $18,8 \%$ (IC95: 17,1\%-20,5\%). Em relação ao estudo das associações, para se trabalhar com erro alfa de 0,05 , erro beta de 0,20 , razão expostos/não expostos de 10/90, e razão de riscos de 1,7, o pre- 
sente estudo deveria incluir pelo menos 1.860 adolescentes. Tal valor já se encontra acrescido de $10 \%$ para perdas e $15 \%$ para controle de fatores de confusão 11 .

A coleta das informações ocorreu por meio de dois questionários padronizados, ambos aplicados no domicílio dos adolescentes. Esses questionários foram preparados com base nas perguntas utilizadas nas pesquisas nacionais sobre demografia e saúde (PNDS), no estudo sobre o aprendizado da sexualidade, reprodução e trajetórias sociais de jovens brasileiros e nos questionários utilizados nas coortes de Pelotas, Rio Grande do Sul. As perguntas foram ainda testadas quanto ao seu enunciado entre adolescentes da mesma região. Quando necessário, alguns termos foram substituídos por aqueles da linguagem local.

O primeiro questionário, mais abrangente, era aplicado pelo entrevistador e buscava informações referentes à família e ao adolescente. Por meio deste questionário investigavam-se características demográficas (cor da pele, idade, sexo e situação marital); condições socioeconômicas (escolaridade, renda familiar); ambientais (condições da residência, área de moradia e aglomeração familiar) e características comportamentais (conhecimento sobre doenças sexualmente transmissíveis e métodos contraceptivos). $\mathrm{O}$ segundo questionário era confidencial e autoaplicável. A sua aplicação ocorria da seguinte maneira: um questionário era entregue em um envelope fechado ao adolescente que, em seguida era por ele aberto, lido, respondido, colocado de volta no envelope, lacrado e devolvido ao entrevistador. O último questionário buscava informações sobre uso de drogas lícitas e ilícitas; idade de início de namoro, de vida sexual, de ocorrência de gravidez e aborto. Por fim, nesse mesmo questionário era avaliada a presença de transtorno mental comum pelo SRQ-20 (Self Reporting Questionaire) ${ }^{12}$. No questionário para o sexo feminino, foi investigada, ainda, a idade de ocorrência da menarca.

Para a realização deste estudo, 14 entrevistadores com segundo grau completo e estudantes de graduação do Campus de São Raimundo Nonato da Universidade Estadual do Piauí (UESPI) foram selecionados e treinados durante quatro dias. Desses, apenas oito participaram do estudo. Durante o treinamento, realizou-se leitura dos questionários e do manual de instruções e simulação de entrevistas. O estudo piloto foi realizado no quinto dia em São Raimundo Nonato e visava testar cada questão.

Os entrevistadores foram divididos em duplas e designados a áreas distintas de cada município, as quais foram anteriormente mapeadas e numeradas. Todos os questionários eram codifi- cados e revisados pelos entrevistadores e duplamente digitados, em ordem inversa e por diferentes digitadores usando o Epi Info 6.04 (Centers for Disease Control and Prevention, Atlanta, Estados Unidos).

O controle de qualidade foi realizado com revisão dos questionários e repetição parcial de 5\% das entrevistas. O objetivo principal dessa etapa era confirmar a realização das entrevistas e comparar as respostas obtidas.

A análise ajustada foi realizada obedecendo ao modelo hierárquico previamente definido com três níveis 13. Mantiveram-se no modelo as variáveis com valor de $\mathrm{p} \leq 0,20$. Como medida de associação calculou-se a razão de prevalências (RP) com intervalo de 95\% de confiança (IC95\%) 14. No primeiro nível, mais distal, foram incluídas as variáveis demográficas dos adolescentes e socioeconômicas da família; no segundo, variáveis relativas à área de moradia e aglomeração familiar; no terceiro, variáveis comportamentais de conhecimento sobre doenças de transmissão sexual e métodos contraceptivos e também referentes ao uso de drogas e à presença de transtorno mental comum. O desfecho foi constituído pelo não conhecimento de preservativo masculino significando "nunca ter ouvido falar" e/ou "nunca tê-lo visto".

É importante salientar que, embora o estudo tivesse coletado informações sobre cerca de 250 variáveis, foram levadas à análise hierarquizada todas as variáveis ditas basais (demográficas, socioeconômicas e ambientais) e aquelas sabidamente associadas ao conhecimento de métodos contraceptivos identificadas em outros estudos. Além dessas, incluíram-se também todas aquelas variáveis relacionadas a hipóteses do estudo previamente definidas quando da elaboração do projeto de pesquisa. Dentro desse contexto, toda variável cujo valor de $\mathrm{p}$ tenha sido $\leq 0,20$ foi incluída para ajuste no modelo de análise previamente determinado.

$\mathrm{Na}$ análise estatística, utilizou-se o qui-quadrado de Pearson para comparar proporções e regressão de Poisson com ajuste robusto da variância para a obtenção da medida de efeito, tanto na análise bruta quanto na ajustada. A heterogeneidade foi avaliada pelo teste de Wald e o nível de significância empregado foi de 95\%. Toda a análise estatística foi conduzida pelo uso do programa Stata 11.2 (Stata Corp., College Station, Estados Unidos).

Somente os adolescentes com 18 anos ou mais de idade é que assinaram o Termo de Consentimento Livre e Esclarecido. Para os demais, o termo era assinado pelos pais. Este projeto de pesquisa foi submetido ao Comitê de Ética em Pesquisa da Faculdade de Medicina da Uni- 
versidade Federal de Pelotas (CEPAS/UFPel) e aprovado. Foi financiado pelo Conselho Nacional de Desenvolvimento Científico e Tecnológico (CNPq), no Edital MCT/CNPq/MS-SCTIEDECIT/CT-SAUDE no 022/2007.

\section{Resultados}

O presente estudo identificou 2.276 moradores com idade entre 13 e 19 anos residentes nos dois municípios. Quatro deles apresentavam limitações cognitivas que os impedia de responder ao questionário, resultando em 2.272 adolescentes elegíveis para o estudo. Desse total, três se recusaram a participar do estudo e 28 não foram encontrados nos seus domicílios mesmo após três tentativas. Assim, o questionário aplicado pelo entrevistador alcançou taxa de respondentes de $98,6 \%$ ( $1,4 \%$ de perda). Todos esses 2.241 adolescentes receberam envelope fechado contendo um questionário autoaplicável. Dentre eles, 245 não foram respondidos na sua quase totalidade, o que revela taxa de resposta de $87,8 \%$. Dentre os 1996 questionários autoaplicáveis respondidos, 99 não o foram para as perguntas relativas ao SRQ-20, o que resultou na exclusão deles da analise final. Logo, para a avaliação do SRQ-20 de transtorno mental comum, as taxa de respondentes foi $81,2 \%$. Vale destacar que todas as taxas de respondentes foram calculadas em relação ao denominador elegível, ou seja, 2.272 adolescentes.

A Tabela 1 tem por objetivo mostrar a prevalência de exposição para diversas variáveis demográficas, socioeconômicas, ambientais e de hábitos de vida para a população adolescente vivendo em região do semiárido. Dados representativos sobre essa população não se encontram facilmente disponíveis na literatura e podem ser úteis a outros pesquisadores por ocasião do cálculo do tamanho amostral de estudos com população semelhante. Esta tabela mostra que a maioria dos adolescentes residia em área rural, com distribuição semelhante conforme o sexo; metade deles possuía até 15 anos de idade e $85 \%$ eram de cor da pele parda. Pouco mais da metade era solteiro e sem namorada; $21 \%$ possuíam nove anos ou mais de escolaridade, tendo completado em média seis anos de escolaridade com aprovação. Em relação à renda, 53\% das famílias recebia até 1 salário mínimo mensal, sendo a renda per capita média mensal de R\$90. Quanto ao conhecimento sobre DST, $10 \%$ disseram não conhecer qualquer uma delas; $60 \%$ disseram conhecer contraceptivo oral e $30 \%$ contraceptivo injetável. Cerca de $70 \%$ dos adolescentes começaram a namorar antes dos 14 anos, e a média de idade na primeira relação sexual foi 15 anos; 53\% de todos eles usam ou já usaram alguma droga lícita e/ ou ilícita; $22 \%$ apresentaram transtorno mental comum. De modo geral, adolescentes de Anísio de Abreu apresentaram melhores condições em relação àqueles de Caracol.

A Tabela 2 mostra a prevalência de não conhecimento de preservativo por categoria da variável incluída no modelo e a análise bruta $\mathrm{e}$ ajustada. A prevalência de não conhecimento variou de $4 \%$ para aqueles com $\geq 9$ anos ou mais de escolaridade a $74 \%$ entre os que não conheciam qualquer DST. Na análise bruta, mostraram-se significativamente associadas ao desfecho as variáveis idade, cor da pele, situação marital, escolaridade, renda familiar, conhecer DST e contraceptivo oral e injetável, idade que começou a namorar e se usa ou usou droga lícita e/ou ilícita. Ajustando-se o desfecho para diversos fatores de confusão, verificou-se que a RP para não conhecimento de preservativo masculino foi de 1,36 (IC95\%: 1,15-1,61) em relação aos adolescentes do sexo masculino; quanto maior a idade menor a RP; solteiros sem namorada apresentaram $\mathrm{RP}=$ 1,68 (IC95\%: 1,35-2,09) em relação àqueles com namorada. Quanto menor a escolaridade, maior o risco para o não conhecimento de preservativo; adolescentes que conhecem três ou mais DST apresentaram RP = 0,22 (IC95\%: 0,11-0,42) comparados aos que não conhecem qualquer uma; a RP foi 0,07 (IC95\%: 0,03-0,13) para adolescentes que conhecem contraceptivo oral em relação aos que não conhecem.

\section{Discussão}

Neste estudo, pertencer ao sexo feminino, possuir menor idade, escolaridade e renda familiar, ser solteiro/a sem namorada/o, desconhecer doenças de transmissão sexual e contraceptivo oral mostraram-se associados de forma independente ao não conhecimento de preservativo masculino.

Dezoito por cento dos adolescentes estudados disseram nunca ter visto ou mesmo nunca ter ouvido falar em preservativo masculino. Essa prevalência é semelhante à encontrada entre adolescentes de 13 a 19 anos, de escolas públicas e privadas no Município de São Paulo, que foi de $17 \%$, mas superior à identificada entre adolescentes de 14 a 17 anos de uma instituição pública de formação profissional em Araçatuba (São Paulo), que foi de $3,0 \% 1$.

Neste estudo, $18,8 \%$ das adolescentes disseram não conhecer preservativo masculino e a RP foi de 1,36 (IC95\%: 1,15-1,61) em relação aos adolescentes do sexo masculino. Em escolas 
Tabela 1

Características socioeconômicas, demográficas e comportamentais dos adolescentes residentes nos municípios de Caracol e Anísio de Abreu, Piauí, Brasil, 2008.

\begin{tabular}{|c|c|c|c|}
\hline \multirow[t]{2}{*}{ Variável } & \multicolumn{2}{|c|}{ Município (\%) } & \multirow[t]{2}{*}{ Total (\%) } \\
\hline & $\begin{array}{c}\text { Caracol } \\
(\mathrm{N}=1.274)\end{array}$ & $\begin{array}{c}\text { Anísio } \\
(\mathrm{N}=967)\end{array}$ & \\
\hline \multicolumn{4}{|l|}{ Área de moradia $(p<0,001)$} \\
\hline Urbana & 37,4 & 48,1 & 42,0 \\
\hline Rural & 62,6 & 51,9 & 58,0 \\
\hline \multicolumn{4}{|l|}{$\operatorname{Sexo}(p=0,360)$} \\
\hline Masculino & 48,8 & 50,8 & 49,7 \\
\hline Feminino & 51,2 & 49,2 & 50,3 \\
\hline \multicolumn{4}{|l|}{ Idade [anos completos] $(p=0,193)$} \\
\hline 13 & 16,3 & 16,6 & 16,5 \\
\hline $14-15$ & 30,8 & 27,4 & 29,1 \\
\hline $16-17$ & 28,8 & 30,7 & 29,6 \\
\hline $18-19$ & 24,1 & 25,3 & 24,6 \\
\hline Média (desvio-padrão) & $15,8(1,9)$ & $15,9(2,0)$ & $15,8(2,0)$ \\
\hline \multicolumn{4}{|l|}{ Cor da pele $(p=0,003)$} \\
\hline Branca & 10,8 & 15,6 & 12,8 \\
\hline Parda/Mulata & 86,9 & 82,4 & 85,0 \\
\hline Preta & 2,3 & 2,0 & 2,2 \\
\hline \multicolumn{4}{|l|}{ Situação marital $(p=0,294)$} \\
\hline Solteiro sem namorada & 51,7 & 55,0 & 53,2 \\
\hline Solteiro com namorada & 37,1 & 34,2 & 35,8 \\
\hline Casado & 11,2 & 10,8 & 11,0 \\
\hline \multicolumn{4}{|l|}{ Escolaridade [anos completos] $(p<0,001)$} \\
\hline $1-4$ & 27,9 & 22,3 & 25,5 \\
\hline $5-6$ & 29,7 & 26,1 & 28,2 \\
\hline $7-8$ & 23,2 & 27,4 & 25,0 \\
\hline 9 ou mais & 19,2 & 24,2 & 21,3 \\
\hline Média (desvio-padrão) & $6,0(2,6)$ & $6,51(2,6)$ & $6,27(2,6)$ \\
\hline \multicolumn{4}{|l|}{ Renda familiar [salários mínimos] $(p=0,654)$} \\
\hline Menos de 1 & 52,5 & 53,9 & 53,1 \\
\hline $1-1,9$ & 29,7 & 27,3 & 28,7 \\
\hline $2-2,9$ & 12,3 & 12,8 & 12,5 \\
\hline 3 ou mais & 5,5 & 6,0 & 5,7 \\
\hline Renda mensal média em R\$ (desvio-padrão) & $492(474)$ & $503(487)$ & $497(479)$ \\
\hline \multicolumn{4}{|l|}{ Número de moradores no domicílio $(p<0,001)$} \\
\hline 1 & 16,5 & 25,1 & 20,2 \\
\hline 2 & 23,1 & 26,6 & 24,6 \\
\hline 3 ou mais & 58,4 & 48,3 & 55,2 \\
\hline Média (desvio-padrão) & $6,3(2,1)$ & $5,6(1,6)$ & $6,0(1,9)$ \\
\hline \multicolumn{4}{|c|}{ Número de doenças de transmissão sexual conhecida pelos adolescentes $(p<0,001)$} \\
\hline 0 & 12,1 & 9,6 & 11,0 \\
\hline 1 & 37,9 & 29,9 & 34,5 \\
\hline 2 & 17,4 & 22,4 & 19,5 \\
\hline 3 ou mais & 32,6 & 38,1 & 35,0 \\
\hline
\end{tabular}

(continua) 
Tabela 1 (continuação)

\begin{tabular}{|c|c|c|c|}
\hline \multirow[t]{2}{*}{ Variável } & \multicolumn{2}{|c|}{ Município (\%) } & \multirow[t]{2}{*}{ Total (\%) } \\
\hline & $\begin{array}{c}\text { Caracol } \\
(\mathrm{N}=1.274)\end{array}$ & $\begin{array}{c}\text { Anísio } \\
(\mathrm{N}=967)\end{array}$ & \\
\hline Conhecem contraceptivo oral $(p=0,011)$ & 64,9 & 59,7 & 62,6 \\
\hline Conhecem contraceptivo injetável $(p<0,001)$ & 30,7 & 23,2 & 27,4 \\
\hline Idade na primeira relação sexual [anos] $(p=0,461)$ & $(n=277)$ & $(n=267)$ & \\
\hline Até 14 & 37,6 & 33,3 & 35,4 \\
\hline $15-16$ & 47,6 & 48,7 & 48,2 \\
\hline $17-19$ & 14,8 & 18,0 & 16,4 \\
\hline Média (desvio-padrão) & $14,8(1,8)$ & $14,9(1,8)$ & $14,9(1,8)$ \\
\hline Idade que começou a namorar [anos] $(p=0,020)$ & $(n=538)$ & $(n=415)$ & \\
\hline Até 12 & 29,6 & 27,7 & 28,8 \\
\hline $13-14$ & 43,5 & 37,1 & 40,7 \\
\hline 15 ou mais & 26,9 & 35,2 & 30,5 \\
\hline Média (desvio-padrão) & $13,5(1,7)$ & $13,7(1,8)$ & $13,6(1,8)$ \\
\hline Usa ou já usou drogas lícitas e ou ilícitas $(p=0,001)$ & $(n=1.080)$ & $(n=863)$ & \\
\hline Sim & 49,8 & 57,5 & 53,2 \\
\hline Apresentaram transtorno mental comum segundo SRQ-20 * $(p=0,097)$ & $(n=1.016)$ & $(n=829)$ & \\
\hline Sim & 23,2 & 20,0 & 21,8 \\
\hline Não conhecem (ou nunca ouviram falar) em preservativo masculino $(p=0,636)$ & 18,4 & 19,3 & 18,8 \\
\hline
\end{tabular}

SRQ-20: Self-Reporting Questionnaire.

* Oito ou mais respostas positivas em 20 possíveis.

Tabela 2

Análise bruta e ajustada para fatores associados ao não conhecimento de preservativo masculino entre adolescentes residentes em Caracol e Anísio de Abreu, Piauí, Brasil, 2008.

\begin{tabular}{|c|c|c|c|}
\hline \multirow[t]{2}{*}{ Nível/Variável } & \multirow{2}{*}{$\begin{array}{l}\text { Não conhecem } \\
\text { preservativo } \\
\text { masculino (\%) }\end{array}$} & \multicolumn{2}{|c|}{$\begin{array}{c}\text { Análise } \\
\text { RP (IC95\%) }\end{array}$} \\
\hline & & Bruta & Ajustada \\
\hline \multicolumn{4}{|l|}{ I } \\
\hline Área de moradia & & $p=0,239$ & $p=0,279$ \\
\hline Urbana & 17,6 & 1,00 & 1,00 \\
\hline Rural & 19,6 & $1,11(0,93-1,33)$ & $0,91(0,77-1,08)$ \\
\hline Sexo & & $p=0,992$ & $p<0,001$ \\
\hline Masculino & 18,8 & 1 & 1 \\
\hline Feminino & 18,8 & $1,00(0,84-1,19)$ & $1,36(1,15-1,61)$ \\
\hline Idade (anos completos) & & $p<0,001$ & $p<0,001$ \\
\hline 13 & 34,0 & 1,00 & 1,00 \\
\hline 14 & 28,0 & $0,82(0,65-1,04)$ & $0,98(0,79-1,22)$ \\
\hline 15 & 20,9 & $0,61(0,48-0,79)$ & $0,92(0,72-1,18)$ \\
\hline $16-17$ & 12,8 & $0,37(0,29-0,48)$ & $0,67(0,52-0,86)$ \\
\hline $18-19$ & 9,4 & $0,27(0,21-0,37)$ & $0,54(0,40-0,74)$ \\
\hline Cor da pele & & $p=0,048$ & $p=0,666$ \\
\hline Branca & 14,2 & 1,00 & 1,00 \\
\hline Parda & 19,3 & $1,35(1,00-1,82)$ & $1,02(0,76-1,35)$ \\
\hline Preta & 26,5 & $1,86(1,08-3,21)$ & $1,24(0,75-2,05)$ \\
\hline
\end{tabular}

(continua) 
Tabela 2 (continuação)

\begin{tabular}{|c|c|c|c|}
\hline \multirow[t]{2}{*}{ Nível/Variável } & \multirow{2}{*}{$\begin{array}{l}\text { Não conhecem } \\
\text { preservativo } \\
\text { masculino (\%) }\end{array}$} & \multicolumn{2}{|c|}{$\begin{array}{c}\text { Análise } \\
\text { RP (IC95\%) }\end{array}$} \\
\hline & & Bruta & Ajustada \\
\hline \multicolumn{4}{|l|}{ I } \\
\hline Situação marital & & $p<0,001$ & $p<0,001$ \\
\hline Solteiro com namorada & 10,8 & 1,00 & 1,00 \\
\hline Solteiro sem namorada & 25,9 & $2,39(1,91-2,97)$ & $1,68(1,35-2,09)$ \\
\hline Casado & 10,5 & $0,97(0,64-1,47)$ & $0,89(0,59-1,35)$ \\
\hline Escolaridade (anos completos) & & $p<0,001$ & $p<0,001$ \\
\hline $1-4$ & 35,8 & 1,00 & 1,00 \\
\hline $5-6$ & 22,2 & $0,62(0,51-0,74)$ & $0,64(0,53-0,76)$ \\
\hline $7-8$ & 10,0 & $0,28(0,21-0,37)$ & $0,31(0,23-0,41)$ \\
\hline 9 ou mais & 4,2 & $0,12(0,07-0,18)$ & $0,17(0,11-0,27)$ \\
\hline Renda familiar em tercis & & $p<0,001$ & $p<0,001$ \\
\hline Primeiro (menor) & 23,7 & 1,00 & 1,00 \\
\hline Segundo & 17,6 & $0,74(0,61-0,91)$ & $0,76(0,63-0,92)$ \\
\hline Terceiro (maior) & 15,0 & $0,64(0,51-0,79)$ & $0,77(0,63-0,94)$ \\
\hline Número de moradores no domicílio & & $p=0,002$ & $p=0,518$ \\
\hline 1 & 14,2 & 1,00 & 1,00 \\
\hline 2 & 16,7 & $1,18(0,88-1,58)$ & $0,92(0,69-1,21)$ \\
\hline 3 & 19,9 & $1,41(1,06-1,87)$ & $1,08(0,83-1,41)$ \\
\hline 4 & 22,6 & $1,59(1,22-2,08)$ & $1,06(0,83-1,36)$ \\
\hline \multicolumn{4}{|l|}{ III } \\
\hline $\begin{array}{l}\text { Número de doenças de transmissão } \\
\text { sexual conhecidas pelos adolescentes }\end{array}$ & & $p<0,001$ & $p<0,001$ \\
\hline 0 & 74,5 & 1,00 & 1,00 \\
\hline 1 & 19,7 & $0,26(0,22-0,31)$ & $0,59(0,40-0,86)$ \\
\hline 2 & 11,6 & $0,16(0,12-0,20)$ & $0,30(0,16-0,55)$ \\
\hline 3 ou mais & 4,3 & $0,06(0,04-0,08)$ & $0,22(0,11-0,42)$ \\
\hline Conhecem contraceptivo oral & & $p<0,001$ & $p<0,001$ \\
\hline Não & 47,2 & 1,00 & 1,00 \\
\hline Sim & 52,8 & $0,04(0,03-0.06)$ & $0,07(0,03-0,13)$ \\
\hline Conhecem contraceptivo injetável & & $p<0,001$ & $p=0,06$ \\
\hline Não & 74,3 & 1,00 & 1,00 \\
\hline $\operatorname{Sim}$ & 25,6 & $0.02(0,01-0,07)$ & $0,14(0,02-1,04)$ \\
\hline Idade na primeira relação sexual (anos) & & $p=0,800$ & $p=0,066$ \\
\hline Até 14 & 7,3 & 1,00 & 1,00 \\
\hline $15-16$ & 5,7 & $0,79(0,39-1,60)$ & $0,68(0,41-1,22)$ \\
\hline $17-19$ & 6,7 & $0,93(0,37-2,34)$ & $1,89(0,47-7,63)$ \\
\hline Idade que começou a namorar (anos) & & $p=0,005$ & $p=0,212$ \\
\hline Até 12 & 13,1 & 1,00 & 1,00 \\
\hline $13-14$ & 12,6 & $0.96(0,64-1,44)$ & $0,68(0,47-0,99)$ \\
\hline 15 ou mais & 5,5 & $0.42(0,24-0,74)$ & $0,68(0,38-1,24)$ \\
\hline Usa ou já usou droga lícita e/ou ilícita & & $p<0,001$ & $p=0,611$ \\
\hline Não & 20,2 & 1,00 & 1,00 \\
\hline Sim & 10,2 & $0,50(0,40-0,63)$ & $1,10(0,76-1,59)$ \\
\hline $\begin{array}{l}\text { Apresentaram transtorno mental } \\
\text { comum pelo SRQ-20* }\end{array}$ & & $p=0,147$ & $p=0,793$ \\
\hline Não & 14,8 & 1,00 & 1,00 \\
\hline Sim & 11,9 & $0,80(0,60-1,08)$ & $0,95(0,67-1,35)$ \\
\hline
\end{tabular}

SRQ-20: Self-Report Questionnaire.

* Oito ou mais respostas positivas. 
agrícolas da UESPI, 8\% das adolescentes afirmaram desconhecer preservativo masculino contra $4 \%$ no sexo masculino, enquanto no estudo do interior de São Paulo, não houve diferença conforme o sexo do entrevistado para adolescentes de 14 a 17 anos de idade 1,5 .

No que diz respeito à idade, $9,4 \%$ dos adolescentes de 18 a 19 anos disseram que não conheciam preservativo contra $34 \%$ daqueles com 13 anos de idade. A RP entre essas categorias foi de 0,54 (IC95\%: 0,40-0,74). Entre adolescentes de escolas públicas e privadas do Município de São Paulo, a RP para o conhecimento de métodos contraceptivos entre os mais velhos foi de 1,10 (IC95\%: 1,02-1,19) em comparação aos mais novos 4 . Esses resultados reforçam o achado deste estudo de que quanto maior é a idade, menor é a probabilidade de o adolescente não conhecer preservativo.

Em relação à escolaridade, este estudo identificou que a prevalência de não conhecimento diminui à medida que aumentam os anos de estudo, variando de $35,8 \%$ entre adolescentes com 1 a 4 anos de escolarização a $4,2 \%$ entre os com 9 ou mais anos concluídos. A RP entre aqueles com maior escolaridade foi de 0,17 (IC95\%: 0,11$0,27)$ em relação aos com menos anos de estudo. Entre escolares do Município de São Paulo, ter maior escolaridade mostrou-se significativamente associado ao conhecimento sobre métodos contraceptivos, sendo a RP entre aqueles com maior escolaridade de 1,51 (IC95\%: 1,17$1,95)$ comparados aos que apresentaram menor tempo de estudo ${ }^{4}$. Dois outros estudos indicam também que o grau de escolaridade é fator de proteção para questões relacionadas à sexualidade e prática sexual 7,15. Em um deles, o risco de engravidar foi $58 \%$ menor para adolescentes com cinco anos ou mais de escolaridade em relação aos demais ?.

Referente à renda familiar, 23,7\% dos adolescentes de menor renda não conheciam preservativo em relação àqueles cujas famílias possuíam renda $\geq 3$ salários mínimos. Tem sido observado que os provenientes de famílias de maior renda têm maior possibilidade de conhecer métodos contraceptivos quando comparados àqueles de menor poder aquisitivo 3,4. Estudos conduzidos com adolescentes de regiões mais vulneráveis do país, como os incluídos nesta análise, ou aqueles que vivem nas áreas mais pobres das grandes cidades, indicam que estes apresentam maiores riscos de desenvolver gravidez precoce e pior conhecimento quanto à sexualidade 6,16.

Quanto à situação marital, este estudo identificou que $26 \%$ dos adolescentes solteiros e sem namorada não conheciam preservativo, contra $10 \%$ dos casados ou solteiros com namorada.
A RP para desconhecimento dentre os solteiros sem namorada foi de 1,68 (IC95\%: 1,35-2,09) em comparação às duas outras categorias. É possível sugerir que a possibilidade de ocorrência de relações sexuais tenha como consequência a busca de algum método, explicando o maior conhecimento daqueles em relacionamento estável em relação aos adolescentes sem namorado/a.

Três quartos dos entrevistados disseram não conhecer qualquer DST. Adolescentes do sexo feminino cadastradas em um Núcleo de Saúde da Família de Ribeirão Preto (São Paulo), mencionaram conhecer entre 5 e 6 DST, sendo o HIV/AIDS citada por todos eles 17 . Isso pode ser explicado pela diferença entre as localidades em termos de nível socioeconômico e acesso a informações. Importante considerar que na faixa etária dos 13 aos 19 anos, houve aumento na taxa de infecção de adolescentes do sexo feminino em relação ao masculino, observando-se feminização do HIV/ AIDS, nessa população, no país 17 .

A RP para o não conhecimento de preservativo masculino entre adolescentes que disseram conhecer contraceptivo oral foi $93 \%$ menor em relação aos que não conhecem esse método contraceptivo. Tal fato sugere que a probabilidade de alguém que conhece algum método vir a conhecer um segundo é muito maior do que aquele que não conhece qualquer método vir a conhecer um deles. Além disso, o adolescente parece mais preocupado com a prevenção da gravidez do que com a contaminação por DST 18.

Em relação à presença de transtorno mental comum, identificou-se neste estudo prevalência de 21,8\%. Estudo realizado em Pelotas, com 970 adolescentes de 15 a 19 anos, encontrou prevalência de 28,8\%. Altas taxas de prevalência indicam que os transtornos mentais na adolescência são bastante relevantes para a saúde pública, estando associados ao sexo feminino, à condição socioeconômica baixa e à pouca escolaridade necessitando, portanto, maior atenção 19,20.

Ao interpretar esses dados é necessário ter em conta a possibilidade de viés de informação em virtude do tema tratado neste estudo e de causalidade reversa, por tratar-se de um estudo com delineamento transversal. O questionamento sobre sexualidade pode causar constrangimento e desconfiança quanto ao sigilo das informações, por ser assunto de foro íntimo. A fim de minimizar essa limitação, os questionários eram anônimos e confidenciais e a participação do adolescente voluntária. No entanto, há de se considerar que esse tipo de limitação afeta a quase totalidade dos estudos que tratam do tema, fato que garante a comparabilidade dos seus resultados com os demais. 
Por fim, os dados aqui apresentados mostram a necessidade de novas ações visando ao aumento do conhecimento desses adolescentes no que diz respeito à contracepção, prevenção de gravidez na adolescência e conhecimento sobre doenças de transmissão sexual. É importante salientar que os mais pobres devem ser entendidos como prioritários à ação em virtude de apresentarem de forma sistemática maior prevalência e os maiores riscos de não conhecimento de preservativo masculino em relação aos demais grupos. Vale destacar ainda que uma parceria entre profissionais das áreas da saúde e da educação poderia ser muito oportuna para alcançar maior proporção de adolescentes com informações capazes de melhorar o conhecimento dessa população sobre contracepção e, dessa forma, evitar situações desagradáveis como, por exemplo, gravidez indesejada e doenças transmitidas pela relação sexual. Isso pode ter repercussões importantes sobre o seu futuro.

\section{Resumen}

Se buscó determinar la prevalencia y factores de riesgo asociados a la falta de conocimiento sobre preservativos entre los adolescentes en dos municipios de la región semiárida de Piauí, Brasil, a mediados de 2008. Entrevistadores formados aplicaron un cuestionario estandarizado a todos los adolescentes (13-19 años) en sus hogares. Se investigaron factores como el demográfico, socioeconómico y el conocimiento sobre anticoncepción y enfermedades de transmisión sexual (ETS). El resultado es que no conocían los preservativos. En el análisis multivariante se utilizó la regresión de Poisson con ajuste robusto de la varianza. De los 2.241 adolescentes, un 18,8\% dijo que no conocía los preservativos. La prevalencia de la falta de conocimiento osciló entre un 4\% y los 9 años de escolaridad o más, al $74 \%$ entre los que no saben acerca de las ETS. En el análisis ajustado, ser mujer, tener poca edad, bajo nivel educativo, carecer de pareja, conocer la anticoncepción oral y las ETS aumenta el riesgo de no conocer los preservativos en relación con otras categorías. Las cifras indican la urgencia de la intervención para los adolescentes de estos municipios.

Condones; Anticoncepción; Enfermedades de Transmisión Sexual; Adolescente

\section{Colaboradores}

J. M. Hartmann participou da revisão de literatura, elaboração do projeto, análise e interpretação dos dados e redação do artigo. J. A. Cesar colaborou na elaboração do projeto, análise e interpretação dos dados e redação da versão final do artigo.

\section{Agradecimentos}

A pesquisa realizada teve apoio do CNPq e das prefeituras dos municípios estudados, Anísio de Abreu e Caracol, Piauí. 


\section{Referências}

1. Garbin CAS, Lima DP, Dossi AP, Arcieri RM, Rovida TAS. Percepção de adolescentes sobre doenças sexualmente transmissíveis e métodos contraceptivos. DST J Bras Doenças Sex Transm 2010; 22:60-3.

2. Departamento de Ações Programáticas Estratégicas, Secretaria de Atenção à Saúde, Ministério da Saúde. Direitos sexuais, direitos reprodutivos e métodos anticoncepcionais. http://portal.sau de.gov.br/portal/arquivos/pdf/cartilha_direitos_ sexuais_2006.pdf (acessado em 20/Jul/2012).

3. Belo MAV, Silva JLP. Conhecimento, atitude e prática sobre métodos anticoncepcionais entre adolescentes gestantes. Rev Saúde Pública 2004; 38:479-87.

4. Martins LBM, Costa-Paiva LHS, Osis MJD, Sousa MH, Pinto-Neto AM, Tadini V. Fatores associados ao uso de preservativo masculino e ao conhecimento sobre DST/AIDS em adolescentes de escolas públicas e privadas do município de São Paulo, Brasil. Cad Saúde Pública 2006; 22:315-23.

5. Mendonça RCM, Araújo TME. Métodos contraceptivos: a prática dos adolescentes das escolas agrícolas da Universidade Federal do Piauí. Esc Ana Nery Rev Enferm 2009; 13:863-71.

6. Fundo das Nações Unidas para a Infância. O direito de ser adolescente: oportunidade para reduzir vulnerabilidades e superar desigualdades. Brasília: Fundo das Nações Unidas para a Infância; 2001.

7. Leite IC, Rodrigues RM, Fonseca MC. Fatores associados com o comportamento sexual e reprodutivo entre adolescentes das regiões Sudeste e Nordeste do Brasil. Cad Saúde Pública 2004; 20:474-81.

8. Mendes SS, Moreira RM, Martins CBG, Sousa SPS, Matos KF. Saberes e atitudes dos adolescentes frente à contracepção. Rev Paul Pediatr 2011; 29:385-91.

9. Almeida MCCA, Aquino EML, Graffikin L, Magnani RJ. Uso de contracepção por adolescentes de escolas públicas na Bahia. Rev Saúde Pública 2003; 37:566-75.

10. Silva IS. Cancer epidemiology: principles and methods. Lyon: World Health Organization/International Agency for Research on Cancer; 1999.
11. Dean AG, Dean JA, Coulombier D, Brendel KA, Smith DC, Burton AH, et al. Epi-Info, version 6: a word processing, database, and statistics program for epidemiology on microcomputers. Atlanta: Centers of Disease Control and Prevention; 2002.

12. Mari JJ, Williams P. A validity study of a psychiatric sreening questionnaire in primary care in the city of São Paulo. Br J Psyquiatry 1986; 148:23-6.

13. Victora CG, Huttly SR, Fuchs SC, Olinto MT. The role of conceptual frameworks in epidemiological analysis: a hierarchical approach. Int J Epidemiol 1997; 26:224-7.

14. Kirkwood BR, Sterne JAC. Essentials of medical statistics. 2nd Ed. London: Blackwell Science; 2003.

15. Duarte CM, Nascimento VB, Akerman M. Gravidez na adolescência e exclusão social: análise de disparidades intraurbanas. Rev Panam Salud Pública 2006; 19:236-43.

16. Gupta N, Leite IC. Tendências e determinantes de fecundidade entre adolescentes no Nordeste do Brasil. Perspectivas Internacionais de Planejamento Familiar 2001; n. esp.:24-9\&45.

17. Departamento de DST, Aids e Hepatites Virais, Ministério da Saúde. Boletim Epidemiológico Aids e DST 2012; Ano IX, no 1.

18. Doreto DT, Vieira EM. O conhecimento sobre doenças sexualmente transmissíveis entre adolescentes de baixa renda em Ribeirão Preto, São Paulo, Brasil. Cad Saúde Pública 2007; 23:2511-6.

19. Costa AG, Ludermir AB. Transtornos mentais comuns e apoio social: estudo em comunidade rural da Zona da Mata de Pernambuco, Brasil. Cad Saúde Pública 2005; 21:73-9.

20. Pinheiro KA, Horta BL, Pinheiro RT, Horta LL, Terres NG, Silva RA. Common mental disorders in adolescents: a population based cross-sectional study. Rev Bras Psiquiatr 2007; 29:241-5.

Recebido em 26/Dez/2012

Versão final reapresentada em 13/Mai/2013

Aprovado em 05/Jun/2013 